\title{
Characterization of Triacylglycerols in Madeira Laurel Oil by HPLC-Atmospheric Pressure Chemical Ionization-MS
}

\author{
Paula C. Castilho ${ }^{a, *}$, Maria do Céu Costa ${ }^{a, b}$, \\ Ana Rodrigues $^{b}$, Pedro Costa Branco ${ }^{b}$, and Mafalda Costa ${ }^{b}$ \\ ${ }^{a}$ Centro de Química da Madeira, Universidade da Madeira, Campus Universitário da Penteada, 9000-390 Funchal, \\ Portugal, and ${ }^{b}$ INETI-Instituto Nacional de Engenharia, Tecnologia e Inovação, 1649-038, Lisboa, Portugal
}

\begin{abstract}
Madeira laurel oil was fractionated by liquid extraction combined with TLC, and TAGs were analyzed by HPLC coupled with atmospheric pressure chemical ionization-MS (APCI-MS). Eluted molecular species compositions of the eluted TAG in the complex natural mixture were determined by GC identification of FAME and by LC-atmospheric pressure chemical ionization (APCI)-MS analysis of the lipid. The APCI-MS spectra of most TAG exhibited $[\mathrm{M}+\mathrm{H}]^{+}$and $[\mathrm{M}-\mathrm{RCOO}]^{+}$ ions, which defined the M.W. and the molecular association of fatty acyl residues, respectively. Despite the relatively high degree of saturation, with a saturated/unsaturated ratio of 0.70 , no totally saturated TAG nor mixed asymmetric TAG with two saturated FA (SSM or SSU, where $S$ is saturated, $M$ is monounsaturated, and $U$ is unsaturated) were found. This type of molecular structure provides a possible explanation for the relatively low m.p. $\left(12-15^{\circ} \mathrm{C}\right)$ and also the high oxidative resistance observed.

Paper no. J10807 in JAOCS 81, 913-919 (October 2004).
\end{abstract}

KEY WORDS: APCI-MS, HPLC, Madeira laurel oil, triacylglycerols.

Until 2002, the Laurus spp. endemic to the Macaronesian archipelagos of the Azores, Madeira, and Canaries were considered as a single species, referred to as Laurus azorica (Seub.) Franco. Recently, it was determined that there were enough distinctions to consider the laurels from the Madeira and Canaries as a separated taxon, now classified as L. novocanariensis (1). For centuries, the oil expressed from the ripe fruits of Madeira laurel has been used in traditional medicine. Madeira laurel oil is mentioned in Draggendorf's 1898 medicinal plant encyclopedia. The diaspora has spread its use to South Africa and Latin America, especially Venezuela, where the community of Madeira descendants actually exceeds the population of the parent island. Recorded uses include oral and external application as an anti-infective preparation, external application as an antirheumatic, and internal consumption for stomach, blood, and bowel problems (2). Anti-inflammatory properties of Madeira laurel have been established by our group (Castilho, P., M.C. Costa, A.I. Rodrigues, and L. Corvo). Interestingly, these traditional uses of laurel oil are absent from the other archipelagos of the Macaronesian region where the tree exists.

*To whom correspondence should be addressed. E-mail: castilho@uma.pt
Nowadays, laurel oil is sold directly by producers and local general stores in rural areas, whereas in urban centers it is available from pharmacies and health-products shops. To our knowledge, there is no study reporting the chemical characterization of this oil.

Like olive oil, Madeira laurel oil is a "natural fruit juice" in the sense that both are obtained from the fruit of a tree solely by mechanical or other physical processes; the berries of $L$. novocanariensis are similar in aspect to wild olives and from them a fatty oil is extracted according to procedures quite similar to primitive olive oil production. Originally, it was mechanically expressed and percolated at room temperature; owing to the increase in demand, coupled with the protective legal restrictions from which the botanical species has been benefiting, the oil is now produced by boiling the berries in water before expression. The method affords higher yields, although it increases the risk of reducing the aroma.

For most vegetable oils, the major compounds are TAG, present in 95-98\% (3,4); in Madeira laurel oil, TAG account for $85 \%$, the other $15 \%$ being composed of hydrocarbons (mainly mono- and sesquiterpenes), lactones, sterols, and wax esters.

Knowledge of TAG structure is important with regard to nutritional functions, quality control, technological characteristics (in particular viscosity and melting temperature), and authenticity establishment (4-6). The exact structural composition of the lipid fraction of the palm and other vegetable oils has been investigated $(7,8) .{ }^{13} \mathrm{C}$ NMR spectroscopy not only supported the identification of the existing FA as determined by GC but also showed that these FA were present almost exclusively as TAG.

HPLC is the most extensively used technique for the analysis of TAG species of oils and fats (9). Atmospheric pressure chemical ionization (APCI), being one of the mildest ionization techniques available (10-12), is particularly suitable for the analysis of TAG with HPLC-MS, owing to the relatively simple mass spectra produced and the possibility of identifying the positional isomers. The $s n-1$ and $s n-3$ positions of TAG cannot be distinguished, although by means of MS, (11), the FA combinations and regioisomeric structure of most ACN/n (acyl carbon number/number of double bonds) species can be rapidly determined with minimal sample preparation, which is not easily achieved by other methods. 


\section{EXPERIMENTAL PROCEDURES}

Materials and methods. Laurel oil was obtained from three different local producers. In one selected case, production was monitored throughout the whole process, from the collection of the laurel berries to storage, boiling, expression, and bottling.

Fractionation. The fixed oil (50 g) was submitted to hydrodistillation to strip off the volatile compounds, using a Clevengertype apparatus. This process yielded the "essential oil" and an odorless residue containing the lipids and other nonvolatile compounds.

The residue was dissolved in $n$-hexane and filtered through activated charcoal to remove chlorophylls. The filtrate was extracted with four 20-mL portions of methanol. The portion insoluble in methanol is called the lipidic fraction: LF.

The combined methanolic extracts were dried in a rotary evaporator under vacuum at room temperature. The residue was dissolved in $10 \mathrm{~mL}$ of acetonitrile and then extracted with $3 \times 10 \mathrm{~mL}$ of hexane to remove fats and waxes. The acetonitrile solution was concentrated in a rotary evaporator under vacuum at room temperature and the residue redissolved in 1 mL methanol (MF: methanolic fraction, containing sterols, alcohols, and sesquiterpene lactones).

${ }^{13}$ C NMR. Samples of laurel oil, without any treatment, were analyzed by ${ }^{13} \mathrm{C}$ NMR spectroscopy (3). Spectra were recorded on a Bruker AMX 300 (Bruker Instruments Inc., Karlsruhe, Germany) operating at $75.47 \mathrm{MHz}$, equipped with a 5-mm QNP probe. Samples of oil were dissolved in $\mathrm{CDCl}_{3}$, with all chemical shifts $(\delta)$ referred to internal tetramethylsilane. The spectra were recorded at room temperature with a 2.36-s acquisition time, a sweep width of $27,777 \mathrm{~Hz}$, and $32 \mathrm{~K}$ acquisition points to yield a digital resolution of $0.509 \mathrm{~Hz} /$ point. A total of 2,048 scans were collected for each sample, with a $45^{\circ}$ excitation pulse and a 1-s relaxation decay. Carbon-13 spectra were recorded with composite phase decoupling and a digital resolution of $0.763 \mathrm{~Hz} / \mathrm{pt}$.

The lipidic fraction, LF, was analyzed using the same experimental conditions.

Determination of FA composition. FA were converted to FAME and determined following the analytical methods described in Regulations EEC/2568/91 and EEC/1429/92 of the European Union Commission for olive oil.

(i) Conversion of FA into FAME. The fixed oil (25 mg) was accurately weighed into a screw-capped tube $(15 \mathrm{~mL})$, and 1.5 $\mathrm{mL}$ of methanolic sodium hydroxide was added, mixed, and heated at $100^{\circ} \mathrm{C}$ for $7 \mathrm{~min}$. After cooling, $2 \mathrm{~mL}$ of boron trifluoride was added and heated at $100^{\circ} \mathrm{C}$ for $5 \mathrm{~min}$. The tube was cooled to $30-40^{\circ} \mathrm{C}$, and $1 \mathrm{~mL}$ of iso-octane was added; the tube was capped and shaken for $30 \mathrm{~s}$. Saturated sodium chloride solution $(5 \mathrm{~mL})$ was immediately added, and the tube was shaken again. The tube contents were allowed to separate, the top (isooctane) layer was removed, and the lower aqueous layer was extracted again with an additional $1 \mathrm{~mL}$ of iso-octane. The two isooctane extracts were combined, dried over anhydrous sodium sulfate, and concentrated to $1 \mathrm{~mL}$ with a stream of nitrogen.

(ii) Analysis of FAME. Analysis of FAME was performed on a Trace 2000 Thermo Quest CE gas chromatograph (Thermo Electron Corp., Alcobendas, Spain) equipped with a DB-23 (J\&W, Rancho Cordova, CA) fused-silica capillary column, $60 \mathrm{~m} \times 0.25 \mathrm{~mm}$ i.d., $0.25-\mu \mathrm{m}$ film thickness. The temperature was programmed to rise from 70 to $195^{\circ} \mathrm{C}$ at a rate of $5^{\circ} \mathrm{C} \mathrm{min}{ }^{-1}$, stand for $10 \mathrm{~min}$, rise to $220^{\circ} \mathrm{C}$ at $5^{\circ} \mathrm{C} \mathrm{min}{ }^{-1}$, stand at that temperature for another $30 \mathrm{~min}$. Injector and FID temperatures were set at 220 and $280^{\circ} \mathrm{C}$, respectively. The sample injection volume was $0.2 \mu \mathrm{L}$, and the carrier gas was helium at a pressure of $70 \mathrm{kPa}$. Quantification was achieved by internal normalization. Correction factors for this column and equipment were used to convert area percentages into mass percentages, determined through the analysis of reference materials certified by MRC [Bureau Communitaire de Reference (BCR), Brussels].

Determination of TAG structure. The lipidic fraction, LF, was analyzed by HPLC-APCI-MS using a Waters Alliance 2695 separation module, with an Waters 2487 dual-wavelength absorbance detector. MS analysis was carried out on a Bruker Esquire 3000 spectrometer, using positive-mode APCI with an ion trap analyzer. The column used was a Hypersil $\mathrm{C}_{18}$ ODS $3.0 \times 250 \mathrm{~mm}$ i.d. (5.0 mm) (Macherey-Nagel, Düren, Germany). The column was kept at room temperature, and a gradient elution was effected by changing the mobile phase composition from acetonitrile/ethanol (90:10) to $12 \%$ acetonitrile in $90 \mathrm{~min}$. The mobile phase flow was $0.7 \mathrm{~mL} / \mathrm{min}$. The samples were dissolved in acetonitrile/propan-2-ol/n-hexane (2:2:1), and $20 \mu \mathrm{L}$ was injected.

\section{RESULTS AND DISCUSSION}

FA composition. Table 1 presents GC data on the determination of the FA composition of Madeira laurel oils from three different producers (L1, L2, L3), as well as the composition of L. nobilis oil, from the literature (13). L1 represents the sample for which production was monitored and was the one chosen for further structural analysis.

Comparison with data from L. nobilis fruit products is inevitable, since L. nobilis LF, mentioned in The Merck Index (14) as "laurel oil," is obtained by methods that are similar to those used with $L$. novocanariensis, although from powdered dry berries.

Published data on the composition of L. nobilis fat show major discrepancies. The SOFA (15) (Seed Oil Fatty Acids) database has 17 entries for L. nobilis from 1965 to 1999 , but no two entries show consistent data; according to Garnier et al. (16), it is composed of $48.1 \%$ lauric acid, $32.3 \%$ oleic acid, $6.2 \%$ palmitic acid, and $18.4 \%$ linoleic acid. Hafizoglu and Reunanen (17), reporting on Turkish laurel berry fat, referred to the existence of $20 \mathrm{FA}$, of which $54 \%$ is lauric acid, and Frega et al. (13) indicated the presence of $58.1 \%$ lauric acid in the fruit endocarp and its total absence from the pericarp/mesocarp; the overall value for the whole fruit was $20 \%$. These last results for the whole dried drupes, extracted with hexane using a Soxhlet apparatus, are comparable with our own results for the fresh fruits of L. nobilis, boiled and pressed (Castilho, P., M.C. Costa, and A.I. Rodriques, unpublished data).

${ }^{13}$ C NMR analysis. The very complex spectrum of whole 
TABLE 1

FA Composition of Madeira Laurel Oil Samples from Three Different Producers (L1, L2, and L3) and Laurus nobilis Literature Data (13)

\begin{tabular}{lrrrc}
\hline FA & L1 & \multicolumn{1}{c}{ L2 } & \multicolumn{1}{c}{ L3 } & L. nobilis \\
\hline Lauric (C12:0) & 18.6 & 16.4 & 14.0 & 20.0 \\
Myristic (C14:0) & 0.7 & 0.7 & 1.9 & 0.8 \\
Palmitic (C16:0) & 20.8 & 19.3 & 22.5 & 14.1 \\
Palmitoleic (C16:1) & 0.4 & 0.4 & 0.7 & - \\
Stearic (C18:0) & 1.1 & 1.1 & 1.4 & 1.0 \\
Oleic (C18:1) & 32.6 & 31.6 & 32.8 & 42.0 \\
Linoleic (C18:2) & 23.5 & 22.1 & 22.3 & 18.0 \\
Linolenic (C18:3) & 1.3 & 1.1 & 1.3 & 1.0 \\
Eicosenoic (C20:1) & 0.5 & 0.5 & 0.5 & - \\
Total & 99.5 & 99.7 & 97.4 & 97.7 \\
$\Sigma$ (Saturated) & 41.2 & 40.2 & 39.8 & 35.9 \\
$\Sigma$ (Unsaturated) & 58.3 & 59.5 & 57.6 & 61.8 \\
\hline
\end{tabular}

laurel oil shows the characteristic signals of TAG and 1,3DAG; 1,2-DAG signals are detectable as traces (Fig. 1). A molar ratio TAG/1,3-DAG of 96:4 can be deduced by comparison with the areas of the signals of glyceride carbons (60-75 ppm) (7). The carbonyl resonance at $176.8 \mathrm{ppm}$ was assigned to FFA, and the two signals at $c a$. $170 \mathrm{ppm}$ were attributed to sesquiterpene lactones. The ${ }^{13} \mathrm{C}$ NMR spectrum of LF shows only the signals of TAG. Although ${ }^{13} \mathrm{C}$ NMR spectroscopy can provide a lot of essential information regarding the structure of
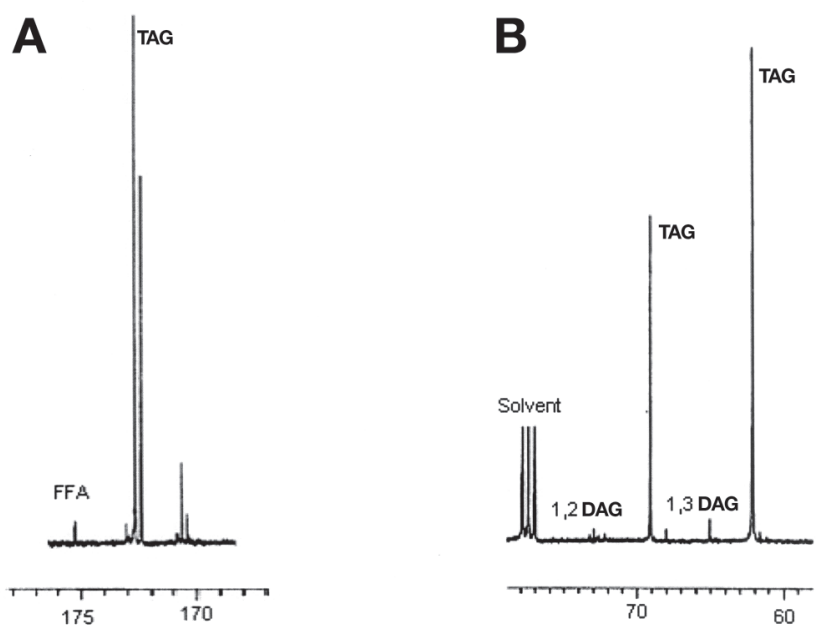

FIG. 1. ${ }^{13} \mathrm{C}$ NMR spectrum $(75.47 \mathrm{MHz})$ of $(\mathrm{A})$ the carbonyl carbons and (B) the glycerol carbons of a sample of Madeira laurel oil.

the acyl groups and their distribution on the glyceride backbone of TAG $(7,8)$, it was used in this study mainly to confirm the identity of the reported FA and to ensure that the obtained LF contained only TAG.

The TAG structure was determined by HPLC-APCI. It is

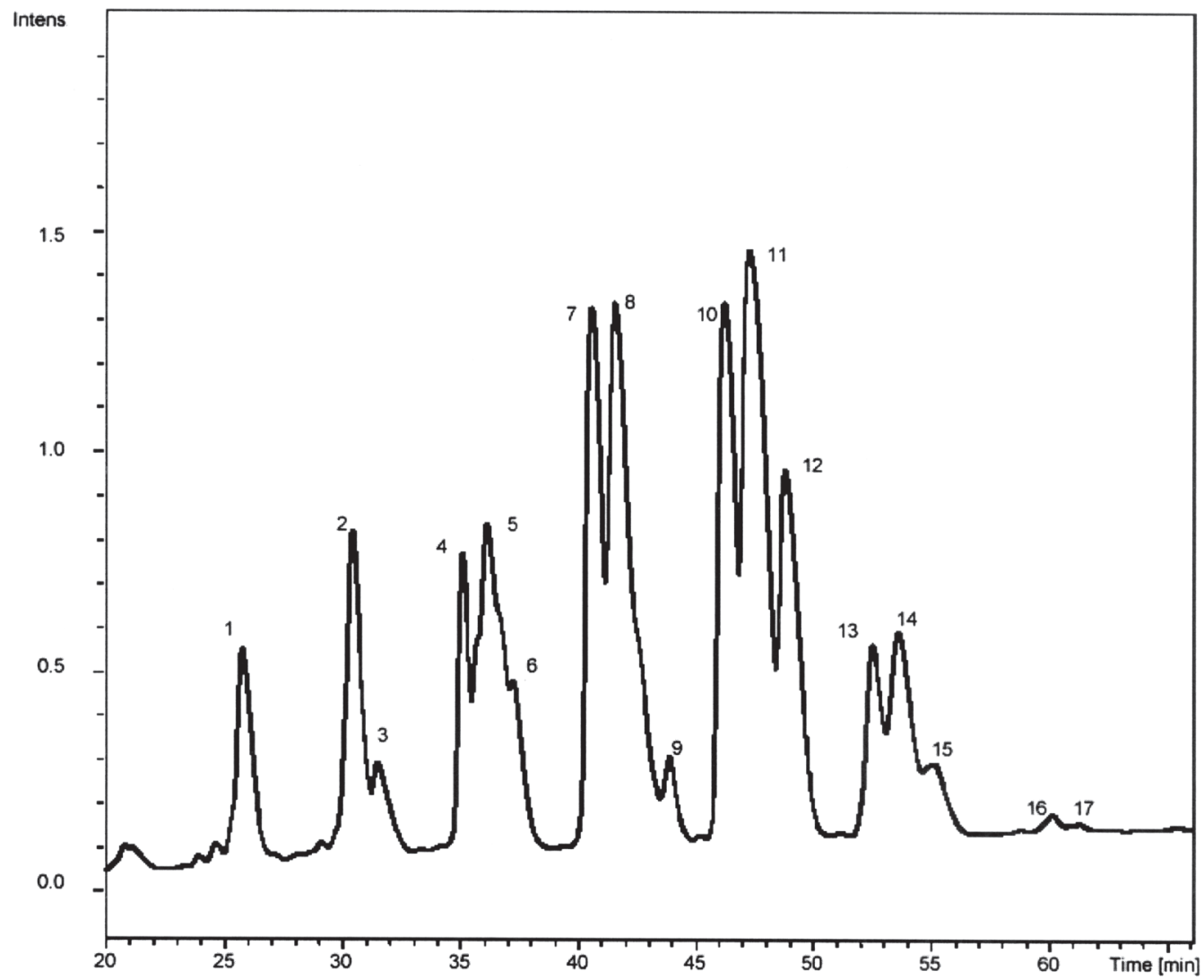

FIG. 2. The reconstructed ion chromatogram (RIC) of TAG from Madeira laurel fruit oil achieved by RP-HPLCAPCl-MS. The assignment of these peaks is presented in Table 2. APCl, atmospheric pressure chemical ionization. 
TABLE 2

Molecular Species Identification ${ }^{a}$ of the TAG of Madeira Laurel Fruit Oil

\begin{tabular}{|c|c|c|c|c|c|c|c|c|c|c|c|}
\hline Peak \# & $\mathrm{RT}$ (min) & TAG & {$[\mathrm{M}+\mathrm{H}]^{+}$} & DAG & {$\left[\mathrm{M}-\mathrm{R}_{1} \mathrm{COO}\right]^{+}$} & DAG & {$\left[\mathrm{M}-\mathrm{R}_{2} \mathrm{COO}\right]^{+}$} & DAG & {$\left[\mathrm{M}-\mathrm{R}_{3} \mathrm{COO}\right]^{+}$} & $\mathrm{ACN}: \mathrm{n}$ & $\mathrm{ECN}$ \\
\hline \multirow[t]{3}{*}{1} & 27.9 & LnLLn & $875.7(20)$ & LLn & $597.5(6)$ & & & & & $54: 8$ & 38 \\
\hline & & LaLLn & 797.6 (8) & & & & & LaL & $519.5(100)$ & $48: 5$ & 38 \\
\hline & & LaLLa & $719.6(3)$ & LaL & 519.5 (100) & LaLa & $439.4(14)$ & & & $42: 2$ & 38 \\
\hline \multirow[t]{2}{*}{2} & 32.7 & LLLn & $877.8(62)$ & & & LLn & $597.5(11)$ & LL & $599.5(56)$ & $54: 7$ & 40 \\
\hline & & LLLa & $799.8(100)$ & LaL & $519.5(32)$ & & & LL & $599.5(56)$ & $48: 4$ & 40 \\
\hline \multirow[t]{2}{*}{3} & 33.8 & LLLn & $877.7(40)$ & & & & & LL & $599.5(57)$ & $54: 7$ & 40 \\
\hline & & LLLa & 799.7 (100) & LaL & $519.5(32)$ & & & & & $48: 4$ & 40 \\
\hline 4 & 37.4 & LLL & $879.9(100)$ & $\mathrm{LL}$ & $599.5(37)$ & & & & & $54: 6$ & 42 \\
\hline \multirow[t]{2}{*}{5} & 38.5 & OLLn & 879.7 (94) & Oln & $599.6(20)$ & LLn & $597.5(13)$ & & & $54: 6$ & 42 \\
\hline & & LOLa & 801.7 (27) & $\mathrm{LaO}$ & $521.5(33)$ & LLa & $519.5(46)$ & $\mathrm{OL}$ & $601.5(100)$ & $48: 3$ & 42 \\
\hline \multirow[t]{4}{*}{6} & 39.7 & OLLn & $879.7(12)$ & OLn & $599.6(9)$ & & & & & $54: 6$ & 42 \\
\hline & & PLLn & $853.8(100)$ & LLn & $597.5(17)$ & PLn & $573.5(12)$ & PL & $575.5(87)$ & $52: 5$ & 42 \\
\hline & & LOLa & $801.6(10)$ & $\mathrm{LaO}$ & $521.4(17)$ & LaL & $519.5(63)$ & $\mathrm{OL}$ & $601.5(38)$ & $48: 3$ & 42 \\
\hline & & PLLa & $775.6(3)$ & PL & $575.5(87)$ & PLa & $495.4(21)$ & LLa & $519.5(63)$ & $46: 2$ & 42 \\
\hline 7 & 42.9 & LOL & $881.9(100)$ & $\mathrm{OL}$ & $601.5(51)$ & & & & & $54: 5$ & 44 \\
\hline \multirow[t]{2}{*}{8} & 44.0 & LOL & $881.8(40)$ & $\mathrm{OL}$ & $601.5(20)$ & & & & & $54: 5$ & 44 \\
\hline & & PLL & $855.6(100)$ & LL & 599.5 (57) & & & $P L$ & $575.5(52)$ & $52: 4$ & 44 \\
\hline \multirow[t]{3}{*}{9} & 46.4 & PLL & 855.7 (65) & LL & $599.5(32)$ & PL & $575.5(94)$ & & & $52: 4$ & 44 \\
\hline & & POLa & & $\mathrm{LaO}$ & $521.5(63)$ & LaP & $495.5(24)$ & $\mathrm{PO}$ & $575.5(94)$ & $46: 1$ & 44 \\
\hline & & PLnP & 829.7 (43) & PLn & $573.5(100)$ & PP & $551.5(26)$ & & & $50: 3$ & 44 \\
\hline 10 & 48.7 & OLO & $883.8(35)$ & OL & $601.7(100)$ & $\mathrm{OO}$ & $603.5(40)$ & & & $54: 4$ & 46 \\
\hline \multirow[t]{2}{*}{11} & 49.8 & OLO & $883.7(11)$ & $\mathrm{OL}$ & $601.6(100)$ & $\mathrm{OO}$ & $603.5(21)$ & & & $54: 4$ & 46 \\
\hline & & PLO & $857.7(24)$ & $\mathrm{OL}$ & 601.6 (100) & $\mathrm{PO}$ & $577.5(51)$ & PL & $575.5(63)$ & $52: 3$ & 46 \\
\hline \multirow[t]{2}{*}{12} & 51.3 & PLO & 857.7 (6) & $\mathrm{OL}$ & $601.6(23)$ & $\mathrm{PO}$ & $577.5(21)$ & & & $52: 3$ & 46 \\
\hline & & PLP & $831.7(2)$ & PL & $575.6(100)$ & PP & $551.5(25)$ & & & $50: 2$ & 46 \\
\hline 13 & 55.5 & OOO & $885.8(8)$ & $\mathrm{OO}$ & 603.7 (100) & & & & & $54: 3$ & 48 \\
\hline \multirow[t]{2}{*}{14} & 56.1 & OOO & 885.7 (5) & OO & 603.6 (100) & & & & & $54: 3$ & 48 \\
\hline & & POO & 859.7 (5) & $\mathrm{OO}$ & $603.6(100)$ & & & $\mathrm{PO}$ & $577.6(80)$ & $52: 2$ & 48 \\
\hline \multirow[t]{2}{*}{15} & 57.6 & POO & 859.7 & $\mathrm{OO}$ & $603.6(49)$ & & & $\mathrm{PO}$ & $577.6(100)$ & $50: 1$ & 48 \\
\hline & & POP & $833.6(1)$ & $\mathrm{PO}$ & $577.6(100)$ & PP & $551.5(15)$ & & & $52: 2$ & 48 \\
\hline \multirow[t]{2}{*}{17} & 62.6 & OOS & $887.8(6)$ & SO & $605.6(100)$ & & & $\mathrm{OO}$ & $603.8(48)$ & $54: 2$ & 50 \\
\hline & & OOE & $913.8(3)$ & $\mathrm{EO}$ & $631.6(21)$ & & & $\mathrm{OO}$ & $603.8(48)$ & $53: 6$ & 50 \\
\hline
\end{tabular}

a Ln, linolenic; L, linoleic; La, lauric; O, oleic; P, palmitic; S, stearic; ACN, acyl carbon number; ECN, equivalent carbon number; RT, retention time.

well documented that the separation of TAG by RP-HPLC is affected by both the combined number of carbon atoms in the acyl chain (ACN: acyl carbon number) and the number of double bonds, $n$, in the molecule (18). In general, the molecules elute in ascending order of ACN, each of the double bonds reducing the retention on the stationary phase (19). This retention time-reducing effect of double bonds depends on the chromatographic system applied. In addition, TAG with the same equivalent carbon number $(\mathrm{ECN})$, calculated as $\mathrm{ECN}=\mathrm{ACN}-$ $2 n$, are usually difficult to separate $(4,5)$.

Although the separation of TAG was achieved, in the present work the critical groups were only partially resolved. Some peaks were a result of co-elution of up to four TAG; although this co-elution did not prevent the possibility of positive identification of TAG, which could be achieved by singleion chromatograms, it hindered accurate quantification.

Figure 2 presents the reconstructed ion chromatogram of the LF of Madeira laurel oil. The identification of the molecular species was mainly based on the mass spectra data, as well as on the elution properties of TAG according to Figure 2. Mass spectra of TAG were obtained from chromatographic peaks. Single-component peaks produced easily interpretable mass spectra of TAG; multicomponent peaks resulted in more complex mass spectra. However, reliable identification was possible in most cases.
The suggested FA combinations are presented in Table 2. The $[\mathrm{M}+\mathrm{H}]^{+}$ion provided M.W. information on TAG, whereas the $[\mathrm{M}-\mathrm{RCOO}]^{+}$fragment ions gave information on the FA residues (18). In most cases, the regiospecific positions of the FA moieties could be judged, even though one protonated molecular ion, $[\mathrm{M}+\mathrm{H}]^{+}$, can represent several isobaric TAG. The intensities of the acylium cation $\left[\mathrm{RCO}^{+}\right.$peaks in the spectra were not significant, and their contribution was not a necessary feature for the identification of TAG.

TAG positional isomers were identified, taking into account a few generally accepted considerations. Seed oils tend to have PUFA in the $s n-2$ position, but relatively little difference can be found between the $s n-1$ and $s n-3$ primary positions, although less abundant FA are often concentrated in the $s n-3$ position (10). Also, the absence of palmitate in the $s n-2$ position is characteristic of plant TAG in general (5). Palmitic acid in vegetable oils is preferentially incorporated into the $s n-1$ and $s n-3$ positions. TAG with more than four sites of unsaturation gave protonated molecular ions, $[\mathrm{M}+\mathrm{H}]^{+}$, as base peaks, with a low abundance of DAG fragment ions, $\left[\mathrm{M}-\mathrm{RCOO}^{+}\right.$; spectra of TAG with fewer than three sites of unsaturation had DAG fragment ions as base peaks, and TAG with three or four unsaturations could have either of these ions as the base peak $(6,20)$.

The positional isomers can be identified from the relative in- 


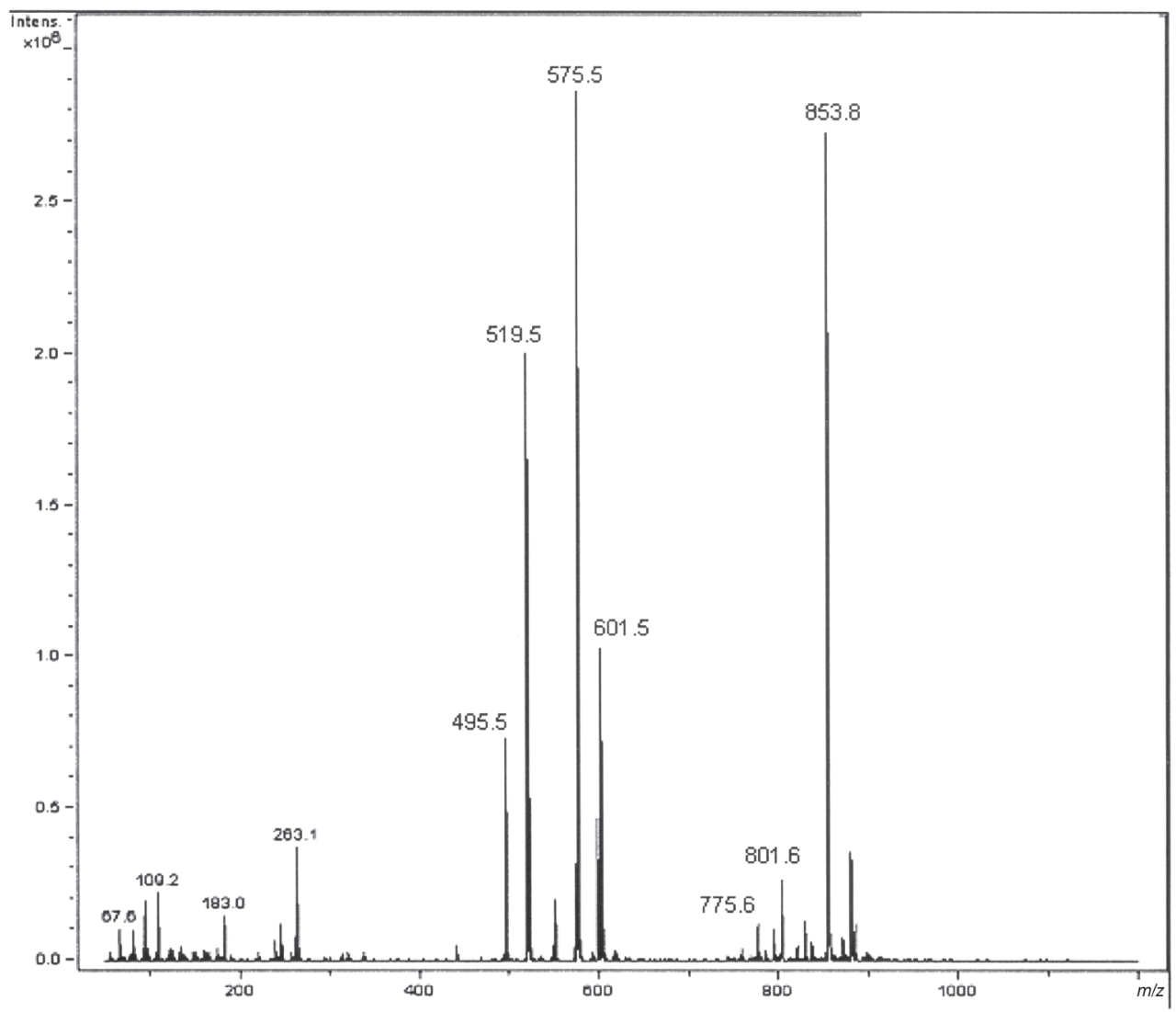

FIG. 3. APCI-mass spectrum of peak 6 (in Fig. 2), corresponding to a mixture of TAG of equivalent carbon number (ECN) 42. For other abbreviation see Figure 2.

tensities of the DAG fragment ions. TAG can be described according to the fragments they produce: (i) AAA type-homogeneous (monoacid) TAG such as OOO and LLL $(\mathrm{O}=$ oleic; $\mathrm{L}=$ linoleic; $\mathrm{P}=$ plamitic; $\mathrm{Ln}=$ linolenic; $\mathrm{Po}=$ palmitoleic); (ii) $\mathrm{ABA}$ type-mixed symmetric TAG containing two different FA such as PLP; (iii) AAB type-mixed asymmetric TAG containing two different FA such as LLLn, LLO, PLL; and (iv) ABC typemixed TAG containing three different FA such as PLO.

For example, in the analysis of ABC-type TAG, the least abundant DAG fragment ion corresponds to the loss of the FA from the $s n-2$ position, since the loss of the FA from the sn- 2 position is the least favorable energetically. The $\mathrm{AAB}$ and $\mathrm{ABA}$ type of TAG also can be distinguished, because the AA/AB ratio of the DAG fragment ions is remarkably different, being much lower than 1 when $\mathrm{B}$ is in the $s n-2$ position $(11,12)$. This is explained on the same basis as the ratio of the DAG fragment ions from ABC-type TAG (8). The least abundant DAG fragment ion is used to identify 2-positional isomers without differentiation between 1- and 3-positions. The position of the FA on the glycerol backbone affects the elution order on the column, with unsaturated FA in the 2-position causing the TAG to elute before the TAG having the same unsaturated FA in the 1or 3-position (11).

As shown in Table 2, the analysis of the chromatographic peaks revealed 21 different TAG having 7 FA moieties. Myristic and palmitoleic acids, identified in the FAME chromato- graphic analysis, were not detected as constituents of these TAG, even though all relevant signals in the mass spectra obtained were accounted for.

Two chromatographic peaks of complex composition deserve a more detailed analysis: peak 6 eluting at $39.7 \mathrm{~min}$, and peak 9 at $46.4 \mathrm{~min}$. Their mass spectra are presented in Figures 3 and 4, respectively. Peak 6 consisted of four TAG of ECN 42. For three of those peaks, both the protonated molecular ion and the DAG ions that could result from its fragmentation were present, allowing the identification: LLL $\rightarrow$ LL $(\mathrm{m} / z$ 599.5); PLLn $\rightarrow \mathrm{PL}(\mathrm{m} / z$ 575.5), LLn $(\mathrm{m} / z$ 597.5), PLn $(\mathrm{m} / z$ 573.5); LOLa $\rightarrow \mathrm{LO}(\mathrm{m} / z$ 601.5), $\mathrm{LaO}(\mathrm{m} / z, 521.4), \mathrm{LaL}(\mathrm{m} / z$ 519.5). The fourth TAG present, PLLa, was inferred based on the $\mathrm{m} / \mathrm{z}$ of peak 495.5, assigned to PLa as a 1,3-DAG fragment, and the presence of a very weak signal at $m / z, 775.6(3 \%)$; having only two double bonds, the relative abundance of the $[\mathrm{M}+\mathrm{H}]^{+}$ should be very small. The other two expected fragments, LLa and PL, were detected as just described. The assumption that these ions resulted from the fragmentation of more than one molecule also explained the relative intensities observed.

Peak 9 is another complex chromatographic peak corresponding to a critical mixture of TAG of ECN 44. PLL, fragmenting into PL and LL, and POLa, fragmenting into OP and $\mathrm{LaP}$, were easily identified. The assignment of $[\mathrm{M}+\mathrm{H}]^{+}$of $\mathrm{m} / \mathrm{z}$ 829.7 to PLnP is not direct. This signal also could be attributed to the TAG PLPo, and the fragment at $\mathrm{m} / z 573.5$ could be due 


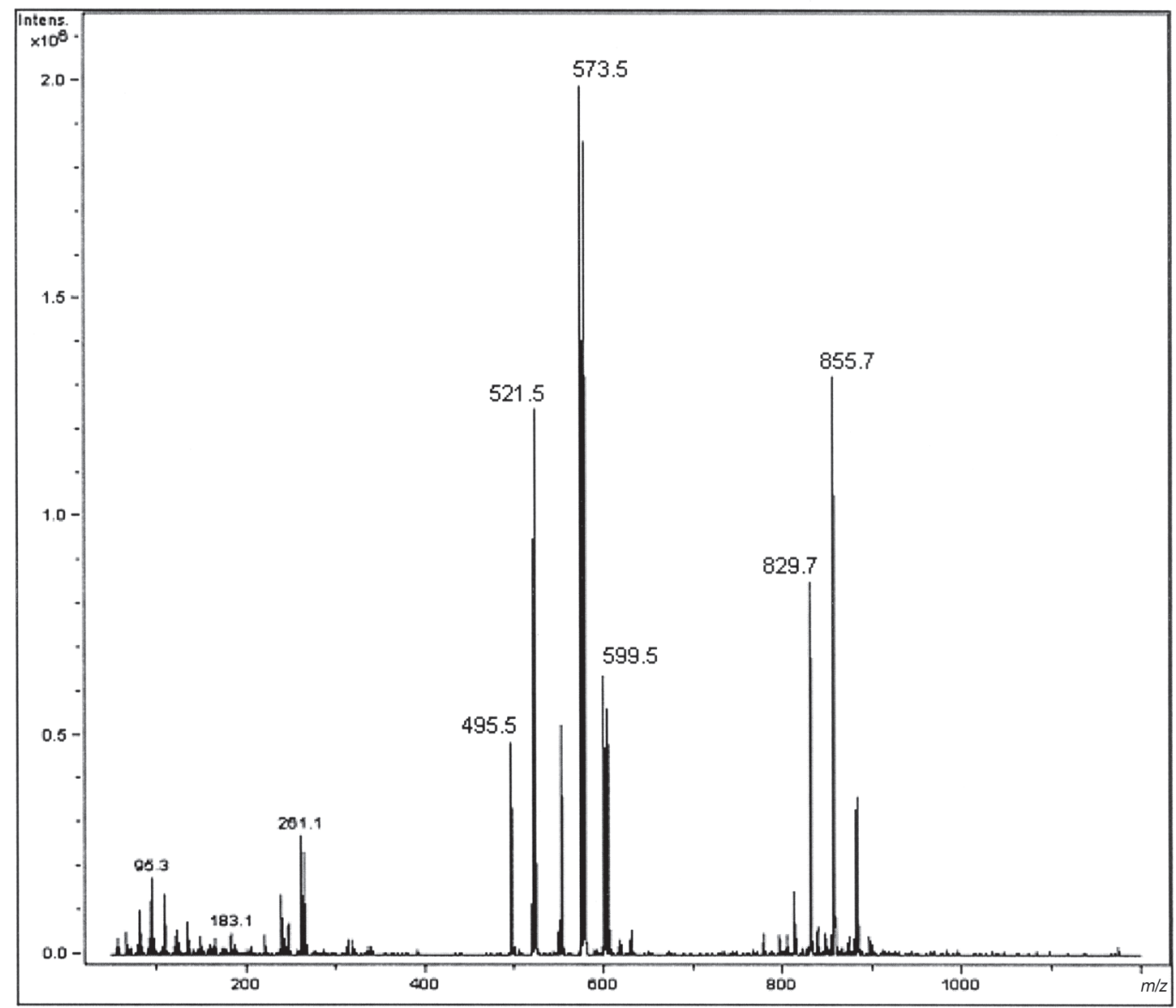

FIG. 4. APCI-mass spectrum of peak 9 (from Fig. 2), corresponding to a mixture of TAG of ECN 44-46. For abbreviations see Figures 2 and 3.

to LPo. However, the fragment PP at $\mathrm{m} / z$ 551.5 ruled out this second assignment, since it could not have originated by the fragmentation of any other co-eluting TAG. The proposal of the symmetric positional isomer was based on the intensity ratio PP/PLn $=0.6$, suggesting that $\mathrm{PP}$ corresponds to the 1,3 DAG fragment.

The four main types of APCI mass spectra were distinguished according to the number and relative intensity of the signals observed: AAA type, monoacid: LLL and OOO; ABA, mixed symmetric type: LaLLa, LnLLn, LOL, OLO, PLP, PLnP, POP; AAB, mixed asymmetric type: LLLn, LLLa, PLL, POO, OOS, OOE; ABC, mixed asymmetric type: LaLLn, LOLa, PLLa, PLLn, POLa, PLO.

Although TAG with the same ECN (critical pairs) were not baseline-resolved, it was possible to establish that the first chromatographic peak of a nonresolved group corresponded to a symmetric TAG (mono- or di-acid) of the major unsaturated FA, oleic and linoleic. Four single-component peaks of similar areas were found, corresponding to LLL, LOL, OLO, and OOO.

Despite the high degree of saturation, with a ratio saturated/unsaturated $=0.70$, no totally saturated TAG $(\mathrm{SSS})$ or mixed asymmetric TAG with two saturated fatty acids (SSM or SSU) were found. This type of structure may explain the relatively low m.p. $\left(12-15^{\circ} \mathrm{C}\right)$, high viscosity, and high oxidative resistance observed for this oil, which does not show changes on ${ }^{13} \mathrm{C}$ NMR or FTIR spectra after 2 yr of storage.

\section{ACKNOWLEDGMENT}

Thanks are due to Dr. Teresa Nogueira, from INETI, for calling the authors' attention to the new taxonomical classification.

\section{REFERENCES}

1. Rivas-Martínez, S., T.E. Díaz, F. Fernández-González, J. Izco, J. Loidi, M. Lousã, and A. Penas, Vascular Plant Communities of Spain and Portugal. Addenda to the Syntaxonomical Checklist of 2001, Part II, Itinera Geobotánica 15:203 (2002).

2. Rivera, D., and C. Obón, The Ethnopharmacology of Madeira and Porto Santo Islands, a Review, J. Pharmacol. 46:73-93 (1995).

3. Cert, A., W. Moreda, and M.C. Pérez-Camino, Chromatographic Analysis of Minor Constituents in Vegetable Oils, $J$. Chromatogr. A 881:131-148 (2000).

4. Andrikopoulos, N.K., Triglyceride Species Compositions of Common Edible Vegetable Oils and Methods Used for Their Identification and Quantification, Food Rev. Int. 18:71-102 (2002). 
5. Kallio, H., K. Yli-Jokipii, J.P. Kurvinen, O. Sjövall, and R. Tahvonen, Regioisomerism of Triacylglycerols in Lard, Tallow, Yolk, Chicken Skin, Palm Oil, Palm Olein, Palm Stearin, and a Transesterified Blend of Palm Stearin and Coconut Oil Analyzed by Tandem Mass Spectrometry, J. Agric. Food Chem. 49: 3363-3369 (2001).

6. Neff, W.E., W.C. Byrdwell, K.R. Steidley, G.R. List, and G. Snowder, Triacylglycerol Structure of Animal Tallows, Potential Food Formulation Fats, by High-Performance Liquid Chromatography Coupled with Mass Spectrometry, J. Liq. Chromatogr. Rel. Technol. 25:985-998 (2002).

7. Ng, S., Quantitative Analysis of Partial Acylglycerols and Free Fatty Acids in Palm Oil by ${ }^{13} \mathrm{C}$ Nuclear Magnetic Resonance Spectroscopy, J. Am. Oil Chem. Soc. 77:749-755 (2000).

8. Zamora, R., and F. J. Hidalgo, Classification of Vegetable Oils by High-Resolution ${ }^{13} \mathrm{C}$ Nuclear Magnetic Resonance Spectroscopy Using Chromatographically Obtained Oil Fractions, Ibid. 79:267-272 (2002).

9. Neff, W.E., and W.C. Byrdwell, Soybean Oil Triacylglycerol Analysis by Reversed-Phase High-Performance Liquid Chromatography Coupled with Atmospheric-Pressure Chemical-Ionization Mass Spectrometry, Ibid. 72:1185-1191 (1995).

10. Kusaka, T., S. Ishihara, M. Sakaida, A. Mifunc, Y. Nakano, K. Tsuda, M. Ikeda, and H. Nakano, Composition Analysis of Normal Plant Triacylglycerols and Hydroperoxidized rac-1Stearoyl-2-oleoyl-3-linoleoyl-sn-glycerols by Liquid Chromatography-Atmospheric Pressure Chemical Ionization Mass Spectrometry, J. Chromatogr. A 730:1-7 (1996).

11. Byrdwell, W.C., Atmospheric Pressure Chemical Ionization Mass Spectrometry for Analysis of Lipids-A Review, Lipids 36:327-346 (2001).
12. Jakab, A., K. Heberger, and E. Forgacs, Comparative Analysis of Different Plant Oils by High-Performance Liquid Chromatography-Atmospheric Pressure Chemical Ionization Mass Spectrometry, J. Chromatogr. A 976:255-263 (2002).

13. Frega, N., L.S. Conte, G. Lercker, and P. Capella, Composizione delle drupe di Laurus nobilis, Riv. Ital. Sostanze Grasse 59: 329-334 (1982).

14. The Merck Index, 12th edn., edited by S. Budavari, M.J. O'Neil, P.E. Heckelman, and J.F. Kinneary, Merck \& Co., Rahway, NJ, 1996.

15. SOFA (Seed Oil Fatty Acids) Database, http://www.bagkf.de/sofa.

16. Garnier, G.L., Bézanger-Beauquesne, and G. Debraux, Ressources Médicinales de la Flore Française, Tome I, Vigot Freres Editeurs, Paris, 1961.

17. Hafizoglu, H., and M. Reunanen, Studies on the Components of Laurus nobilis from Turkey with Special Reference to Laurel Berry Fat, Fett Wissen. Technol. 95:304-308 (1993).

18. Manninen, P., and P. Laakso, Capillary Supercritical Fluid Chromatography Atmospheric Pressure Chemical Ionization Mass Spectrometry of Triacylglycerols in Berry Oils, J. Am. Oil Chem. Soc. 74:1089-1098 (1997).

19. Christie, W.W., The Analysis of Evening Primrose Oil, Ind. Crops Prod. 10:73-83 (1999).

20. Sandra, P., A. Medvedovici, Y. Zhao, and F. David, Characterization of Triglycerides in Vegetable Oils by Silver-Ion Packed-Column Supercritical Fluid Chromatography Coupled to Mass Spectroscopy with Atmospheric Pressure Chemical Ionization and Coordination Ion Spray, J. Chromatogr. A 974:231-241 (2002).

[Received February 20, 2004; accepted September 30, 2004] 